Supplement of Biogeosciences, 17, 5129-5148, 2020

https://doi.org/10.5194/bg-17-5129-2020-supplement

(C) Author(s) 2020. This work is distributed under

the Creative Commons Attribution 4.0 License.

(c) (1)

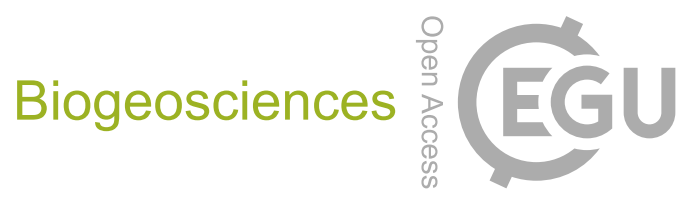

Supplement of

\title{
Nitrogen cycling in CMIP6 land surface models: progress and limitations
}

Taraka Davies-Barnard et al.

Correspondence to: Taraka Davies-Barnard (t.davies-barnard@exeter.ac.uk)

The copyright of individual parts of the supplement might differ from the CC BY 4.0 License. 


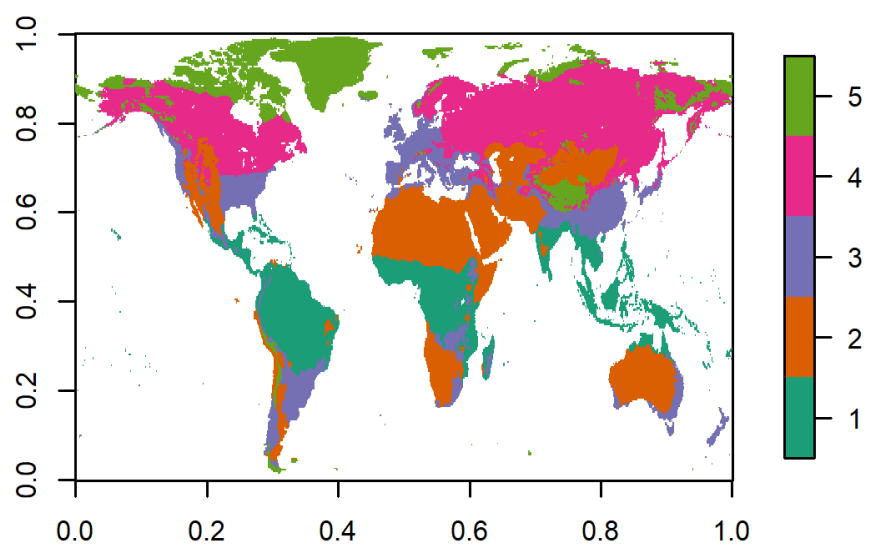

Figure S1. Climate region areas acting as a proxy for biomes, from to Köppen-Geiger climate classification (Kottek et al., 2006; Rohli et al., 2015). 1 = equatorial (tropical), $2=$ arid, $3=$ warm temperate, $4=$ snow (boreal) $5=$ polar (tundra). 

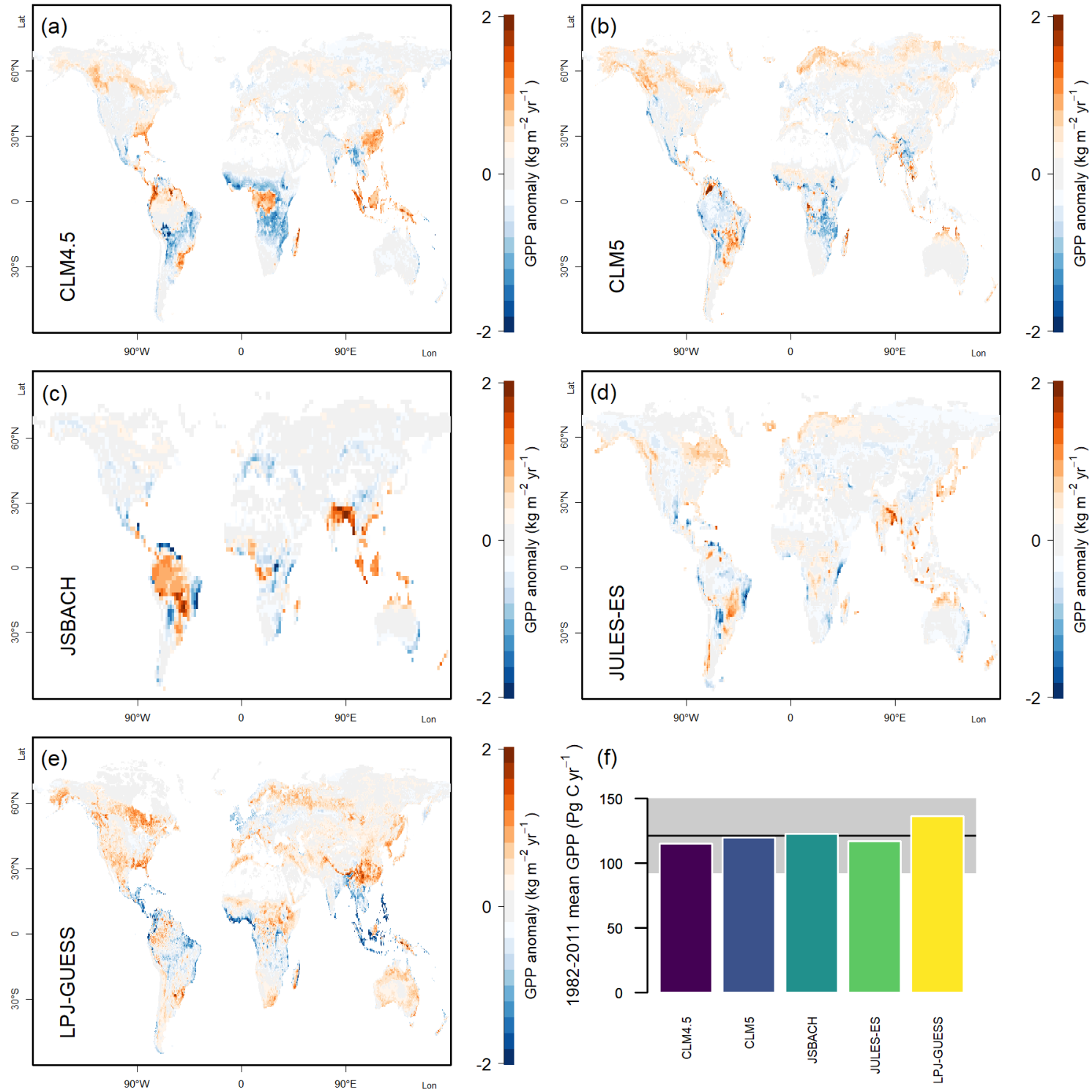

Figure S2. Model output of 1982-2011 mean gross primary productivity (GPP). (a) - (e) Model estimates, shown as the anomaly of the corresponding observation-based estimate (MTE) (i.e. model minus observations) published by Jung et al. (2011). (f) Globally integrated estimates. Black line indicates the global average from the observation-based source; grey area indicates the globally integrated standard deviation from the global average in the model tree ensemble applied to obtain the global average. 


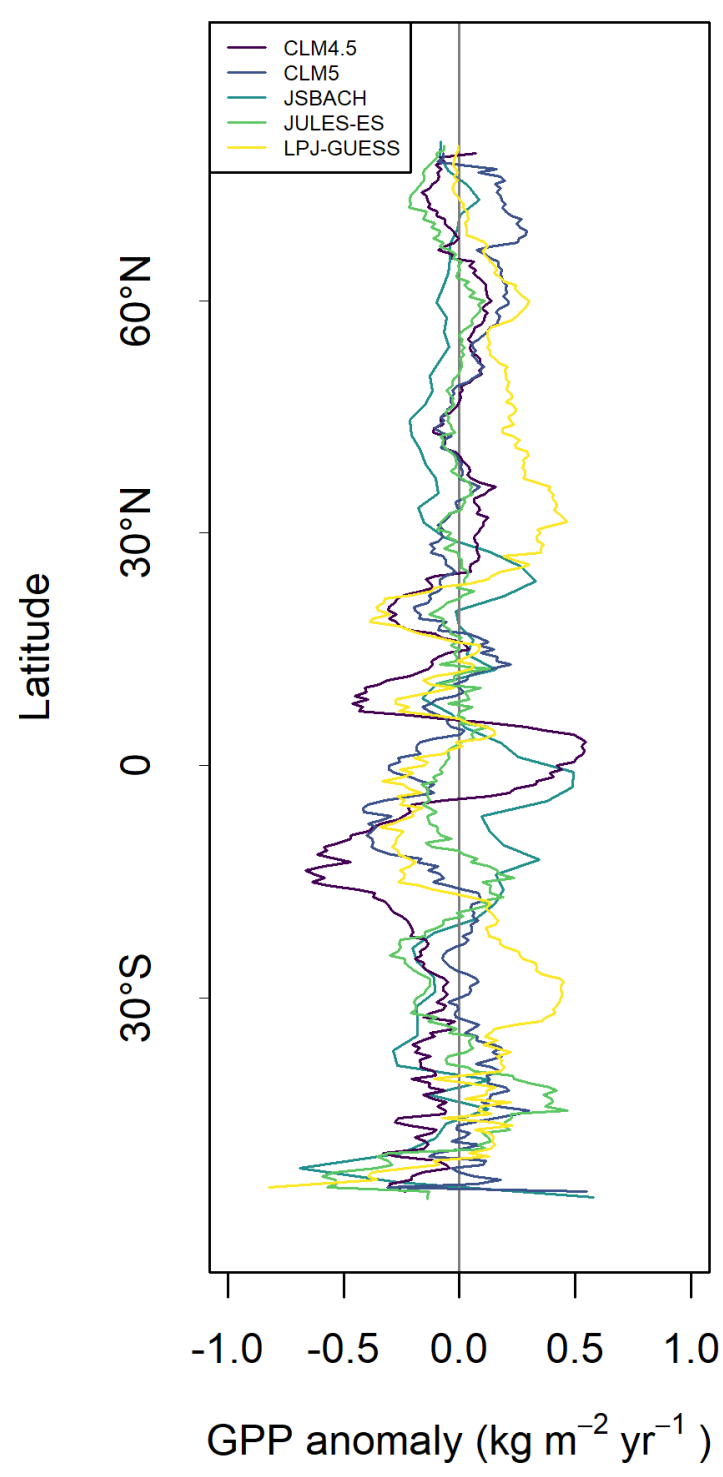

Figure S3. Model output of 1982-2011 mean gross primary productivity (GPP) averaged by latitude, shown as the anomaly of the corresponding observation-based estimate (MTE) published by Jung et al. (2011). 


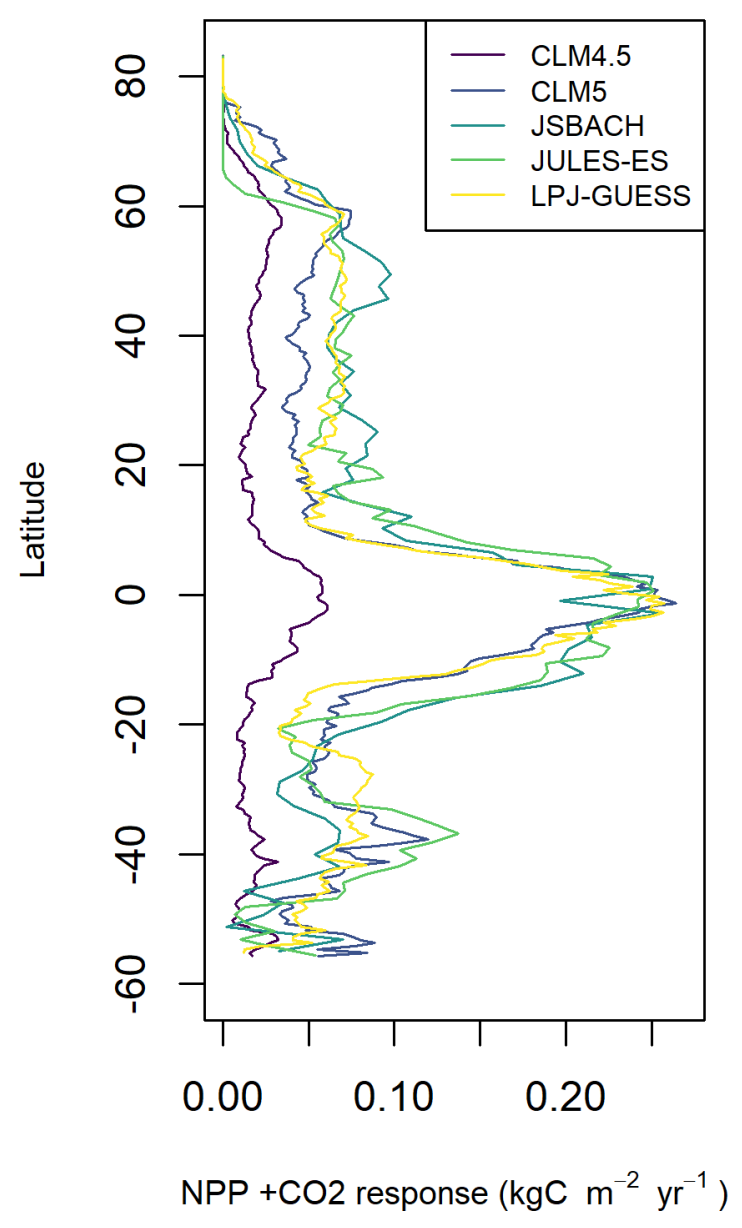

Figure S4. Model estimates of 1996-2005 mean NPP response to +CO2, averaged by latitude. 


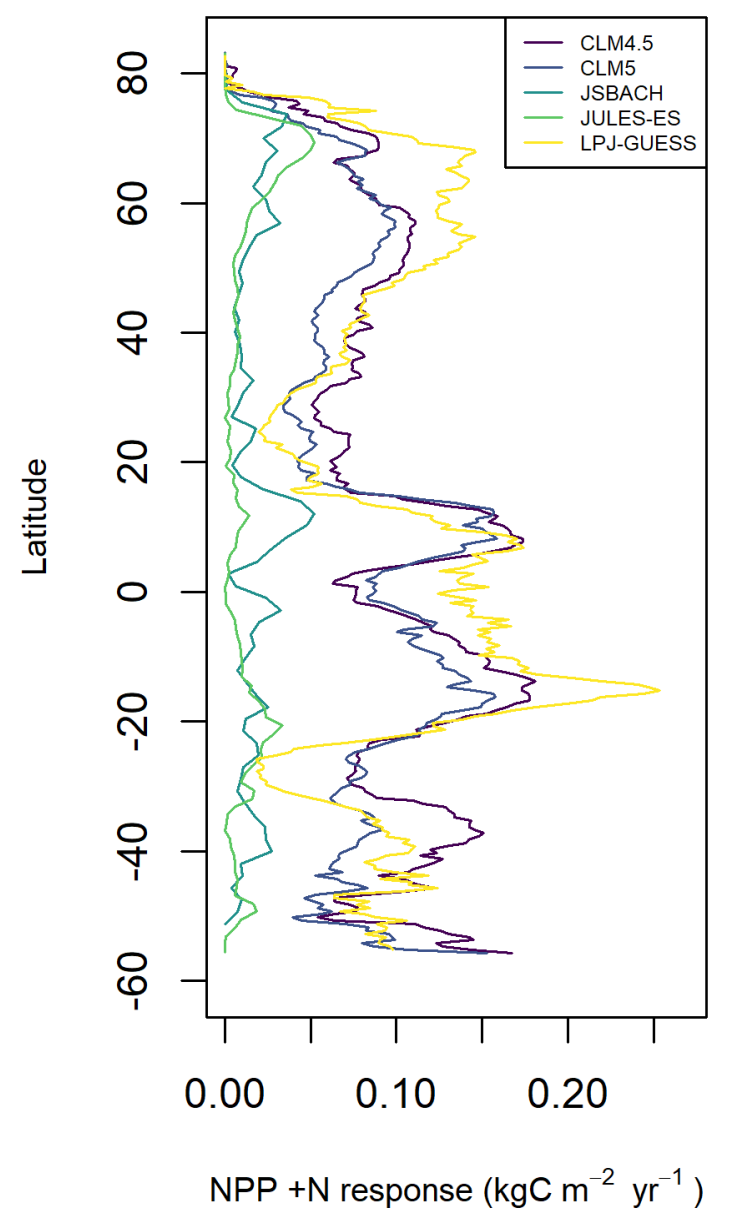

Figure S5. Model estimates of $1996-2005$ mean NPP response to $+\mathbf{N}$, averaged by latitude. 

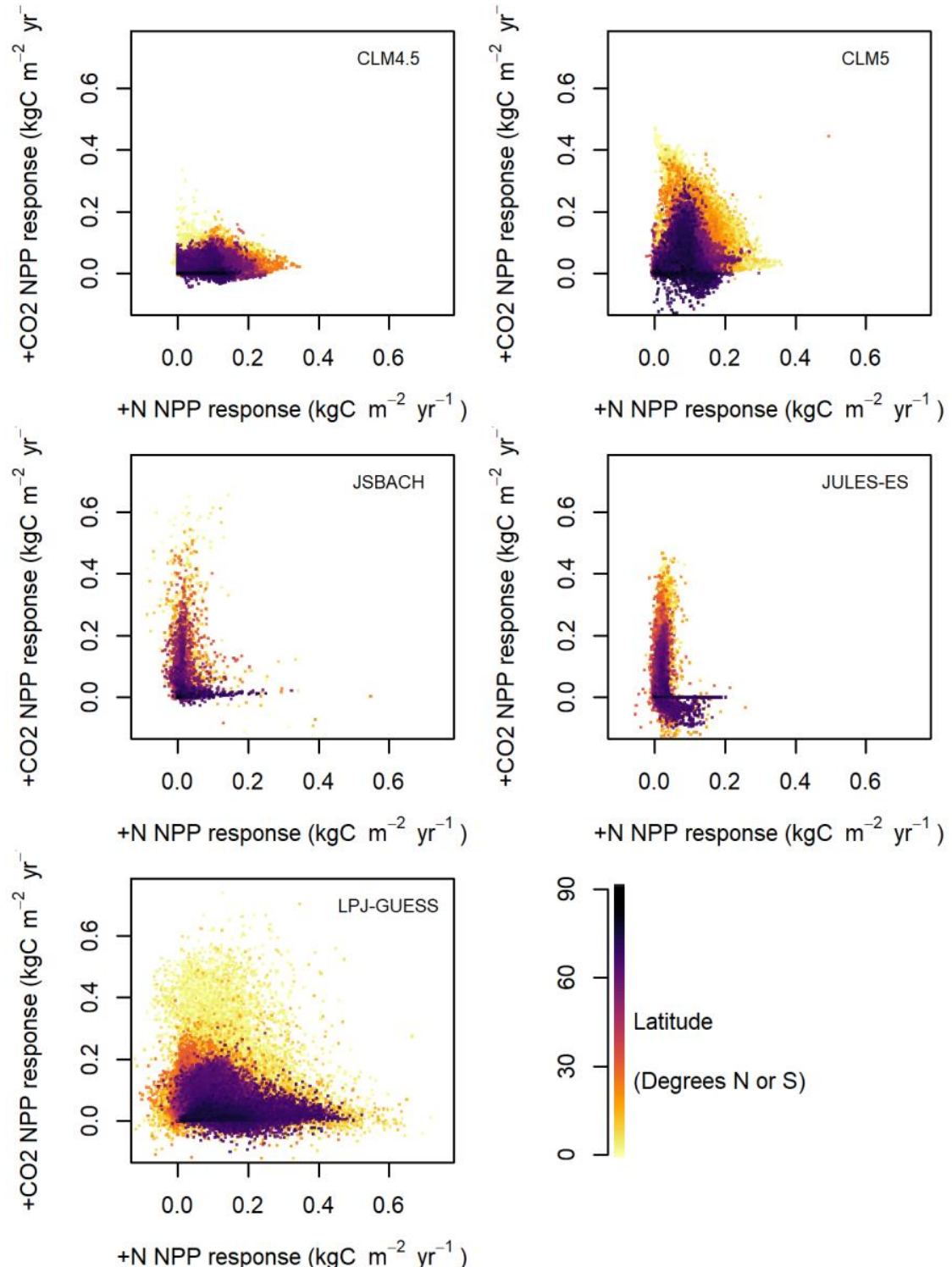

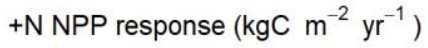

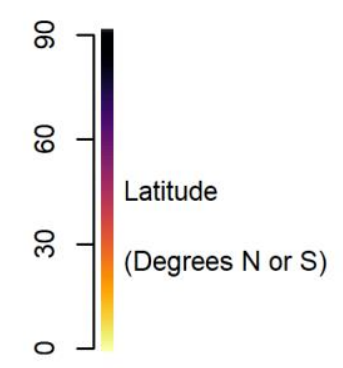

Figure S6. Model estimates of $1996-2005$ mean net primary productivity (NPP) response to $+\mathrm{N}$ vs $+\mathrm{CO2}$ as the anomaly of the control scenario. Each grid box is plotted against the corresponding grid box for the other variable. The colour of the points indicates the latitude, either North or South.

Table S1. Summary of model simulations.

\begin{tabular}{|l|l|l|l|}
\hline & Control & $+\mathbf{C O 2}$ & $+\mathbf{N}$ \\
\hline Atmospheric $\mathbf{C O}_{2}$ & Time varying & Time varying $+200 \mathrm{ppm}$ & Time varying \\
\hline $\mathbf{N}$ deposition & ACCMIP & ACCMIP & $\begin{array}{l}\text { ACCMIP }+50 \mathrm{~kg} \mathrm{~N} \mathrm{ha}^{-1} \mathrm{yr}^{-} \\
1\end{array}$ \\
\hline
\end{tabular}




\begin{tabular}{|c|c|c|c|}
\hline Variable/effect & Dataset & Reference & $\begin{array}{l}\text { Number of } \\
\text { measurements }\end{array}$ \\
\hline \multirow[t]{2}{*}{ +CO2 effect on NPP } & $\begin{array}{l}\text { meta-analysis of total above ground biomass } \\
\text { of woody plants }\end{array}$ & Baig et al., (2015) & 16 \\
\hline & $\begin{array}{l}\text { meta-analysis for whole plant NPP and } \\
\text { aboveground NPP (ANPP) }\end{array}$ & $\begin{array}{l}\text { Song et al., } \\
\text { (2019) }\end{array}$ & $\begin{array}{l}\text { unspecified, maximum } \\
\text { of } 103\end{array}$ \\
\hline \multirow[t]{2}{*}{$+\mathrm{N}$ effect on NPP } & meta-analysis on NPP changes & $\begin{array}{l}\text { LeBauer and } \\
\text { Treseder, (2008) }\end{array}$ & $\begin{array}{l}\text { 126, incl. tundra }(10), \\
\text { tropics }(8), \text { arid land }(3)\end{array}$ \\
\hline & $\begin{array}{l}\text { meta-analysis for whole plant NPP and } \\
\text { aboveground NPP (ANPP) }\end{array}$ & $\begin{array}{l}\text { Song et al., } \\
(2019)\end{array}$ & $\begin{array}{l}\text { unspecified, maximum } \\
\text { of } 429\end{array}$ \\
\hline $\begin{array}{l}\mathrm{BNF} \text { responses to } \\
+\mathrm{CO} 2\end{array}$ & global meta-analysis estimate & $\begin{array}{l}\text { Liang et al. } \\
\text { (2016). }\end{array}$ & 89 \\
\hline BNF responses to $+\mathrm{N}$ & meta-analysis & $\begin{array}{l}\text { Zheng et al., } \\
\text { (2019), }\end{array}$ & $\begin{array}{l}\text { tropical forest }(92), \\
\text { temperate forest }(52) \text {, } \\
\text { boreal forest }(37)\end{array}$ \\
\hline $\begin{array}{l}\text { Biomass response to } \\
+\mathrm{N}\end{array}$ & $\begin{array}{l}\text { aboveground forest biomass } \mathrm{C} \text { change per } \\
\text { added } \mathrm{N} \text { from meta-analysis }\end{array}$ & $\begin{array}{l}\text { Schulte-Uebbing } \\
\text { and Vries, (2018) }\end{array}$ & $\begin{array}{l}\text { tropical }(17), \text { temperate } \\
(41), \text { boreal }(12)\end{array}$ \\
\hline GPP (SI Fig. 2) & Flux tower data model tree ensemble & Jung et al., (2011) & unknown \\
\hline $\begin{array}{l}\text { Biome allocation (SI } \\
\text { Fig. 1) }\end{array}$ & Köppen-Geiger climate classification & $\begin{array}{l}\text { Kottek et al., } \\
2006)\end{array}$ & $\mathrm{n} / \mathrm{a}$ \\
\hline
\end{tabular}

Table S3. Percent $(\%)$ change in mean NPP from $+\mathrm{N}$ or + CO2. Areas where the Control scenario NPP is less than 100 $\mathrm{gC} \mathrm{m}^{-2} \mathrm{yr}^{-1}$ are excluded as the very high percent changes from these grid boxes skew the analysis. Climate regions refer to those shown in SI Fig. 1.

\begin{tabular}{|c|c|c|c|c|c|c|c|c|c|c|}
\hline & \multicolumn{2}{|c|}{ CLM4.5 } & \multicolumn{2}{|l|}{ CLM5 } & \multicolumn{2}{|c|}{ JSBACH } & \multicolumn{2}{|c|}{ JULES } & \multicolumn{2}{|c|}{ LPJ-GUESS } \\
\hline & $+\mathrm{CO2}$ & $+\mathbf{N}$ & $+\mathrm{CO} 2$ & $+\mathbf{N}$ & $+\mathrm{CO2}$ & $+\mathbf{N}$ & $+\mathrm{CO2}$ & $+\mathbf{N}$ & $+\mathrm{CO2}$ & $+\mathbf{N}$ \\
\hline $\begin{array}{l}\text { Equatorial } \\
\text { (Tropical) }\end{array}$ & 4.9 & 17.1 & 22.7 & 16.8 & 17.1 & 1.4 & 19.5 & 0.4 & 18.6 & 19.4 \\
\hline Arid & 6.7 & 52.3 & 17.2 & 63.2 & 31.7 & 9.1 & 7.7 & 5.8 & 17.3 & 14.3 \\
\hline $\begin{array}{l}\text { Warm } \\
\text { Temperate }\end{array}$ & 5.1 & 24.1 & 19.3 & 19.3 & 23.7 & 2.1 & 18.5 & 0.8 & 20.3 & 19.7 \\
\hline $\begin{array}{l}\text { Snow } \\
\text { (Boreal) }\end{array}$ & 6.6 & 28.5 & 15.4 & 23.9 & 18.6 & 3.8 & 13.4 & 3.1 & 14.5 & 27.8 \\
\hline $\begin{array}{l}\text { Polar } \\
\text { (Tundra) }\end{array}$ & 4.4 & 58.4 & 14.0 & 28.1 & 10.7 & 7.9 & 4.1 & 13.2 & 13.7 & 58.7 \\
\hline Global & 5.4 & 24.1 & 19.6 & 22.1 & 19.3 & 2.5 & 16.7 & 1.8 & 17.5 & 21.7 \\
\hline
\end{tabular}

